\title{
Abstract
}

\section{Development of a quantitative PCR assay to evaluate HER2 status of Gastric carcinoma in a cohort of Sri Lankan patients}

Kannangara DKS ${ }^{1}$, Lokuhetty MDS $^{2}$, Dassanayake RS $^{3}$, Gunawardena $\mathrm{NS}^{2}$, Subasinghe $\mathrm{D}^{4}$

${ }^{1} \mathrm{DH}$, Piliyandala, ${ }^{2}$ Faculty of Medicine, Colombo, ${ }^{3}$ Faculty of Medicine, Kelaniya, ${ }^{4}$ National Hospital of Sri Lanka

Key words: quantitative PCR assay, HER2 status, Gastric carcinoma

\begin{abstract}
Introduction
Human epidermal growth factor receptor2(HER2) protein over expression and/or HER2gene amplification are/is linked to dismal outcome of Gastric carcinoma(GCa). Immunohistochemistry $(\mathrm{IHC})$ and fluorescence in situ hybridization(FISH) are keymethods to identify patients for HER2 targeted therapy. Drawbacks of both methods warrant novel-tests.

Objective

To determine whether quantitative Polymerase Chain Reaction (qPCR) could serve as a supplementary-method to evaluate HER2 status of $\mathrm{GCa}$ in a cohort of Sri Lankan patients and investigate correlation between HER2 assessed by different methods and clinicopathological features.

Methods

Twenty GCa-patients with known IHC-HER2 scores were evaluated. qPCR was performed for HER2 gene and Ameloid precursor protein(reference gene) in Formalin fixed paraffin embedded GCa tissue. Threshold values $(\mathrm{Ct})$ were analyzed using Pfaffl-method to detect HER2 gene amplification.

Results

HER2positivity by IHC(protein) and qPCR(gene) were $20 \%$ and $35 \%$ respectively. Sensitivity and specificity of qPCR was $67 \%$ and $76 \%$ respectively and results were reproducible. HER2 protein positivity was correlated with Tumour TNM-stage and Lauren-histological types $(P<0.05)$. Positive expression of HER2 gene was correlated with depth of tumour invasion, differentiation and Lymphnodestatus $(P<0.05)$.Diagnostic consistency between $\mathrm{IHC}$ and $\mathrm{qPCR}(\mathrm{K}=0.146)$ were slightly agreeable $(0.01<k<0.20)$, having $65 \%$ concordance.

Discussion \& Conclusion

Discrepancies between HER2 positivity by IHC and qPCR were possibly due to transcription activation by other genes in the absence of HER2 gene amplification and the aberrant form of HER2 protein not detected by IHC. Studies also indicate a higher-proportion of IHC negative, but HER2gene amplified GCa by FISH.

qPCR may be used as a supplementary-method for detection of HER2 status of GCa in local setting.
\end{abstract}

